\title{
Dévotion populaire et encadrement clérical en Nouvelle-France : la croyance au miracle dans la région de Québec
}

\section{Marie-Aimée Cliche}

Volume 52, 1985

URI : https://id.erudit.org/iderudit/1007000ar

DOI : https://doi.org/10.7202/1007000ar

Aller au sommaire du numéro

Éditeur(s)

Les Éditions Historia Ecclesiæ Catholicæ Canadensis Inc.

ISSN

0318-6172 (imprimé)

1927-7067 (numérique)

Découvrir la revue

Citer cet article

Cliche, M.-A. (1985). Dévotion populaire et encadrement clérical en Nouvelle-France : la croyance au miracle dans la région de Québec. Sessions d'étude - Société canadienne d'histoire de l'Église catholique, 52, 17-34. https://doi.org/10.7202/1007000ar
Résumé de l'article

Le domaine de la croyance au miracle, en Nouvelle-France, offre un champ d'étude privilégié pour comparer les comportements populaires et

l'enseignement de l'Église sur ce sujet. Il constitue un phénomène important dans la vie religieuse de la colonie, notamment dans la région de Québec.
Tous droits réservés @ Les Éditions Historia Ecclesiæ Catholicæ Canadensis Inc., 1985
Ce document est protégé par la loi sur le droit d'auteur. L'utilisation des services d'Érudit (y compris la reproduction) est assujettie à sa politique d'utilisation que vous pouvez consulter en ligne. 


\title{
Dévotion populaire et encadrement clérical en Nouvelle-France: \\ la croyance au miracle dans la région de Québec
}

\author{
Marie-Aimée Cliche \\ Université Laval \\ RÉSUMÉ
}

Le domaine de la croyance au miracle, en Nouvelle-France, offre un champ d'étude privilégié pour comparer les comportements populaires et l'enseignement de l'Église sur ce sujet. Il constitue un phénomène important dans la vie religieuse de la colonie, notamment dans la région de Québec.

Cet exposé sur la croyance au miracle se situe dans le contexte d'une étude sur la religion populaire dans le gouvernement de Québec sous le Régime français ${ }^{1}$. Cet aspect de l'histoire religieuse a retenu l'attention d'un grand nombre de chercheurs au cours des quinze dernières années et suscité des polémiques fameuses autour de la définition du concept de religion populaire ${ }^{2}$. Pour les besoins de la présente analyse, nous utiliserons

\footnotetext{
${ }^{1}$ Ce texte est tiré de notre thèse de doctorat La religion populaire dans le gouvernement du Québec sous le Régime français d'après la pratique des actes surérogatoires, Université Laval, 1984, pp. 39-96.

2 François-A. Isambert, «Religion populaire, sociologie, histoire et folklore», Archives des Sciences sociales des religions (désormais $A S S R$ ), 43,7 (avril-juin 1977), pp. 161-184; 46,1
} 
ce terme controversé pour désigner le comportement religieux de la population laïque, compte tenu des particularités qui peuvent se présenter selon les sexes, les milieux géographiques et les classes sociales.

L'étude de la religion populaire a souvent été abordée en termes de dialectique entre le prescrit et le vécu ${ }^{3}$. Certains auteurs ont beaucoup insisté sur l'opposition entre l'enseignement dispensé par le clergé, surtout celui de la Contre-Réforme, et la religion populaire: le premier, désireux d'instruire les fidèles et de leur inculquer une dévotion théocentrique; la seconde, plus sensible que savante, soucieuse avant tout de secours matériels et cantonnée dans le culte des saints. Jean Delumeau a même parlé d'une volonté d'acculturation de la part du clergé ${ }^{4}$. Toutefois, à mesure que leurs recherches progressaient, les historiens français en sont venus à constater que le clergé post-tridentin avait dû consentir à de nombreux compromis, notamment dans le domaine du culte des saints et des prières de recours matériels, tandis que le peuple assimilait les nouvelles dévotions qu'on lui proposait. Delumeau a constaté, en Bretagne, «une sorte d'accommodement par distorsion du christianisme et des mentalités populaires ${ }^{5}$ tandis qu'Alain Lottin parle d'un mariage difficile mais réussi entre les deux 6 .

En Nouvelle-France, où le clergé a également appliqué les recommandations du concile de Trente, le domaine de la croyance au miracle offre un champ d'étude privilégié pour comparer les comportements populaires et l'enseignement de l'Église sur ce sujet. En effet, c'est autour du culte des saints, de l'utilisation des reliques et des demandes de biens matériels que se produisent les manifestations les plus spontanées des sentiments religieux du peuple. D'autre part, les positions du clergé en ce domaine sont très claires et imprégnées de l'esprit post-tridentin. Enfin, les récits de miracles ${ }^{7}$ sont assez nombreux pour prouver l'importance de ce phénomène dans la vie religieuse de la colonie et permettre d'en faire une

(juillet-septembre 1978), pp. 111-133.

3 Jean Delumeau, «Déchristianisation ou nouveau modèle de christianisme», ASSR, 40 (1975), p. 9.

${ }^{4}$ lbid., p. 12.

5 Jean Delumeau, dir., Histoire du diocèse de Rennes, Paris, Beauchesne, 1979, p. 156.

${ }^{6}$ Alain Lottin, «Contre-Réforme et religion populaire: un mariage difficile mais réussi aux $\mathrm{XVI}^{\mathrm{e}}$ et XVII ${ }^{\mathrm{e}}$ siècles en Flandre et en Hainaut?» dans La Religion populaire, Paris, Éd. du CNRS, 1979, p. 58.

7 Par «miracles», nous entendons les événements extraordinaires que les gens attribuaient à des interventions célestes. Notre intention n'est pas de discuter le bien-fondé de la croyance au miracle ni de prévenir le jugement de l'Église en employant ce terme. Les habitants de la Nouvelle-France croyaient au miracle et considéraient certains événements comme miraculeux. C'est ce fait historique que nous voulons étudier. 
étude quantitative. Cette dernière aura pour but l'analyse systématique des différents aspects du processus miraculeux pour comprendre le mieux possible le comportement des laïcs, celui du clergé, et faire ressortir, par comparaison avec la France, les traits originaux de cet aspect de la religion populaire en Nouvelle-France.

\section{Un élan populaire spontané}

En 1727, à l'Hôpital-Général de Québec, on exhuma les corps de trois religieuses pour les placer dans un nouveau caveau. On s'aperçut alors du bon état de conservation de ces cadavres. La nouvelle se répandit bientôt dans la ville de Québec qu'il s'agissait là d'un fait prodigieux, et une foule de gens se précipita au monastère. "Ils seraient entrés de force» ${ }^{8}$ si on avait tenté de les repousser, écrit la narratrice. Des malades s'y firent transporter. Plus de 30 se déclarèrent guéris, affirme un témoin. Près de 200, renchérit un autre ${ }^{9}$, transporté d'enthousiasme. Une telle manifestation de confiance populaire envers des défunts considérés comme des saints n'était pas exceptionnelle en Nouvelle-France. À la mort de $\mathbf{M}^{\mathrm{gr}}$ de Laval, en 1708, le peuple se pressait autour de sa dépouille pour la mettre en contact avec des objets de piété et le bruit courut bientôt que des guérisons miraculeuses avaient résulté de ces attouchements ${ }^{10}$.

Ces «bruits qui courent» jouaient un rôle important dans la diffusion de la réputation d'un thaumaturge. $\mathbf{M}^{\mathrm{gr}}$ de Lauberivière qui mourut 12 jours après son arrivée au Canada n'avait pas eu beaucoup de temps pour asseoir sa réputation de sainteté parmi la population. Néanmoins, l'information se transmit de bouche à oreille que pendant sa traversée de l'Atlantique, il avait miraculeusement sauvé un enfant tombé à la mer, et cela incita d'autres parents à lui demander la guérison de leur progéniture $^{11}$.

Dans tous ces cas, nous sommes en présence de mouvements populaires spontanés qui ne doivent rien, au point de départ, aux incitations du clergé. Les religieuses de l'Hôpital-Général n'avaient pas invité la population de Québec à envahir leur monastère et la supérieure de l'Hôtel-Dieu se

\footnotetext{
8 Annales de l'Hôpital-Général (manuscrit), II, pp. 50-51, Archives de l'Hôpital-Général de Québec (désormais AHGQ).

9 «Copie d'une lettre de Mère Saint-Augustin Duchesnay écrite à nos mères de France à l'occasion de l'exhumation de trois de nos religieuses en 1727», AHGंQ.

$10 \mathrm{~J}$.-M. Fortier, «La survie de $\mathrm{M}^{\mathrm{gr}}$ de Laval et sa cause de béatification», Société canadienne d' histoire de l'Église catholique, 1957-58, pp. 80 et 82.

${ }^{11}$ Cyprien Tanguay, Monseigneur de Lauberivière, Documents annotés, Montréal, Senécal, 1885 , p. 118.
} 
plaignait de l'importunité des visiteurs qui réclamaient des objets à l'usage de Catherine de Saint-Augustin pour s'en faire des reliques ${ }^{12}$. Mais aussitôt que le phénomène prenait une certaine ampleur, le clergé s'empressait de l'encadrer.

\section{Un clergé attentif}

Face au protestantisme qui niait le bien-fondé du culte des saints, de l'utilisation des reliques et du recours aux miracles, le concile de Trente avait réaffirmé l'enseignement traditionnel de l'Église en cette matière, présentant les miracles comme une manifestation de la toute-puissance divine et du pouvoir d'intercession des saints. Toutefois, pour prévenir les critiques des protestants qui tournaient en dérision l'excès de crédulité des catholiques, les évêques post-tridentins instaurèrent des commissions chargées d'enquêter sur les faits «que le public croit et dit» ${ }^{13}$ miraculeux, comme les guérisons attribuées au frère Didace et à $\mathrm{M}^{\mathrm{gr}}$ de Lauberivière. Quand les récits de miracles semblaient dignes de foi, le clergé les publiait volontiers dans le but d'augmenter la piété du peuple: ce fut le cas pour les miracles arrivés à Sainte-Anne-du-Petit-Cap.

De cette ligne de conduite adoptée par le clergé, il résulte que la plupart des faits miraculeux constatés sous le Régime français ont été relatés par des prêtres et des religieuses. On ne s'étonnera donc pas si ces derniers semblent omniprésents dans le processus miraculeux, que ce soit comme instigateurs de miracles, bénéficiaires ou témoins. Autre conséquence de la nature de ces sources: le récit du miracle se conforme presque toujours parfaitement à l'enseignement de l'Église. Dieu y apparaît habituellement comme l'auteur du prodige, le saint comme un simple intermédiaire, les objets de piété comme des moyens de manifester sa dévotion. Les croyances du peuple pouvaient-elles être aussi parfaitement orthodoxes? Nous ne pouvons les rejoindre qu'à travers l'écran de la phraséologie cléricale. Compte tenu de ces limites, il demeure possible de scruter ces récits pour y discerner la part respective du comportement populaire et de l'interprétation faite par les clercs.

\footnotetext{
${ }^{12}$ Relation des Jésuites (1668), (désormais RJ), Éd. du Jour, t. 6, p. 37.

${ }^{13} \mathrm{C}$. Tanguay, $M^{g r}$ De Lauberivière, p. 129.
} 


\section{L'analyse d'un comportement}

\section{A. Les bénéficiaires des miracles}

Un dépouillement exhaustif des sources imprimées et manuscrites de la région de Québec a permis de retracer 144 récits de miracles concernant des individus ou des groupes restreints de personnes. Parmi les 154 «miraculés», les hommes figuraient en plus grand nombre que les femmes (90 en regard de 60), mais cela dépendait uniquement d'une forte présence des premiers dans les cas de sauvetages maritimes. Les guérisons miraculeuses, par contre, concernaient à peu près également les représentants des deux sexes.

\section{Tableau 1}

Répartition des miraculés selon leur sexe et le type de miracles

$\begin{array}{lccc} & \text { Masculin } & \text { Féminin } & \text { Inconnu } \\ \text { Sauvetages maritimes } & 37 & 8 & 0 \\ \text { Guérisons } & 44 & 48 & 4\end{array}$

Dans les cas où le milieu social des miraculés est connu, deux groupes sont nettement surreprésentés: celui des gens d'Église et celui des notables $^{14}$.

14 Dans la catégorie des notables, nous plaçons les administrateurs, les hauts fonctionnaires, les officiers de justice, les détenteurs de titres honorifiques, les seigneurs, les hommes d'affaires, les gens des services, les capitaines de navire et les officiers militaires. Dans les gens du peuple nous incluons les soldats, les cultivateurs, les gens de métier, les marins et les habitants. Les femmes et les enfants sont classés d'après le statut du chef de famille. 


\section{Tableau 2}

\section{Milieu socio-professionnel des miraculés}

Prêtres et religieuses

$24 \quad 15.6 \%$

Notables

37

$25 \%$

Gens du peuple

$40 \quad 26 \%$

Milieu inconnu

$53 \quad 34,4 \%$

Total

\section{Tableau 3}

Répartition des miracles selon les thaumaturges

\begin{tabular}{|c|c|c|c|}
\hline $\begin{array}{l}\text { Nombre de } \\
\text { miracles }\end{array}$ & Thaumaturge & Types de miracle & Moyens de recours \\
\hline 54 & Sainte Anne & $\begin{array}{l}34 \text { guérisons } \\
20 \text { sauvetages en mer }\end{array}$ & $\begin{array}{l}\text { pèlerinage: } \\
\text { voeu: } \\
\text { messe: } \\
\text { neuvaine: } \\
\text { voeu: } \\
\text { prière: }\end{array}$ \\
\hline 22 & Frère Didace Pelletier & $\begin{array}{l}21 \text { guérisons } \\
1 \text { sauvetage }\end{array}$ & $\begin{array}{l}\text { pèlerinage: } \\
\text { neuvaine: } \\
\text { voeu: } \\
\text { relique: } \\
\text { messe: } \\
\text { prière: }\end{array}$ \\
\hline 17 & Sainte Vierge & $\begin{array}{l}8 \text { guérisons } \\
2 \text { sauvetages en mer } \\
2 \text { incendies } \\
1 \text { bonne mort } \\
1 \text { secours monétaire } \\
1 \text { évasion ( } 2 \text { personnes) } \\
1 \text { secours à un vieillard } \\
1 \text { conversion }\end{array}$ & $\begin{array}{l}\text { voeu: } \\
\text { prière: } \\
\text { image: } \\
\text { pèlerinage: }\end{array}$ \\
\hline 8 & Saint Joseph & $\begin{array}{l}2 \text { bonnes morts } \\
3 \text { secours matériels } \\
1 \text { incendie } \\
1 \text { sauvetage en mer } \\
1 \text { guérison }\end{array}$ & $\begin{array}{l}\text { voeu: } \\
\text { prière: }\end{array}$ \\
\hline
\end{tabular}




\section{Tableau 3 (suite)}

\begin{tabular}{|c|c|c|c|c|}
\hline Nombre de & Thaumaturge & Types de miracle & Moyens de reco & \\
\hline 7 & $\mathrm{M}^{\mathrm{gr}}$ de Lauberivière & $\begin{array}{l}1 \text { sauvetage en mer } \\
6 \text { guérisons }\end{array}$ & $\begin{array}{l}\text { prière: } \\
\text { relique touchée: } \\
\text { pèlerinage: } \\
\text { neuvaine: } \\
\text { voeu: }\end{array}$ & $\begin{array}{l}2 \\
1 \\
2 \\
3 \\
2\end{array}$ \\
\hline 6 & Père Jean de Brébeuf & $\begin{array}{l}5 \text { guérisons } \\
1 \text { conversion }\end{array}$ & $\begin{array}{l}\text { relique bue: } \\
\text { relique touchée: } \\
\text { voeu: }\end{array}$ & $\begin{array}{l}2 \\
1 \\
1\end{array}$ \\
\hline 6 & Dieu ou Christ & $\begin{array}{l}3 \text { guérisons } \\
3 \text { incendies }\end{array}$ & prière & \\
\hline 5 & Sainte Famille & $\begin{array}{l}3 \text { guérisons } \\
1 \text { sauvetage } \\
1 \text { garde d'enfants }\end{array}$ & $\begin{array}{l}\text { voeu: } \\
\text { prière: } \\
\text { médaille: }\end{array}$ & $\begin{array}{l}1 \\
3 \\
1\end{array}$ \\
\hline 4 & Catherine Tekakouita & 4 guérisons & $\begin{array}{l}\text { neuvaine: } \\
\text { voeu: } \\
\text { image: }\end{array}$ & $\begin{array}{l}2 \\
2 \\
1\end{array}$ \\
\hline 3 & Jeanne Leber & 3 guérisons & & \\
\hline 3 & $\begin{array}{l}\text { Catherine de Saint- } \\
\text { Augustin }\end{array}$ & $\begin{array}{l}2 \text { guérisons } \\
1 \text { objet retrouvé }\end{array}$ & $\begin{array}{l}\text { voeu: } \\
\text { neuvaine: } \\
\text { relique touchée: }\end{array}$ & $\begin{array}{l}1 \\
2 \\
1\end{array}$ \\
\hline 2 & Saint François-Régis & 2 guérisons & $\begin{array}{l}\text { neuvaine: } \\
\text { poussière: } \\
\text { image: } \\
\text { prière: }\end{array}$ & $\begin{array}{l}1 \\
1 \\
1 \\
1\end{array}$ \\
\hline 2 & Saint François-Xavier & 2 guérisons & prière & \\
\hline 2 & Saint Michel et anges & 2 guérisons & $\begin{array}{l}\text { voeu: } \\
\text { image: }\end{array}$ & $\begin{array}{l}1 \\
1\end{array}$ \\
\hline 2 & $\mathbf{M}^{\mathrm{gr}}$ de Laval & 2 guérisons & $\begin{array}{l}\text { relique: } \\
\text { attouchement: }\end{array}$ & $\begin{array}{l}1 \\
1\end{array}$ \\
\hline 1 & Saint Augustin & 1 sauvetage en mer & voeu: & 1 \\
\hline 1 & Saint Ignace & 1 guérison & & \\
\hline 1 & Madeleine de Pazy & 1 guérison & voeu: & 1 \\
\hline Imprécis & Père Jacques Marquette & $\begin{array}{l}\text { sauvetages sur le lac } \\
\text { Michigan }\end{array}$ & & \\
\hline Imprécis & $\begin{array}{l}\text { Père Jacques de } \\
\text { Lamberville }\end{array}$ & plusieurs miracles & relique & \\
\hline Imprécis & $\begin{array}{l}\text { D.A.R. Thaumur de La } \\
\text { Source }\end{array}$ & plusieurs miracles & relique & \\
\hline Imprécis & $\begin{array}{l}\text { Trois religieuses de } \\
\text { l'Hôpital-Général }\end{array}$ & plusieurs guérisons & & \\
\hline
\end{tabular}


La proportion élevée de prêtres et de religieuses peut refléter une plus grande piété de leur part, mais elle dépend aussi de la nature des sources: les chroniqueurs des communautés religieuses laissaient rarement passer l'occasion de faire connaître un miracle qui s'était produit sous leur toit. Il est possible également que les religieux et les personnes placés aux plus hauts échelons de la société aient été plus conscients que les autres de la nécessité de rendre les miracles publics pour hâter la canonisation de leur auteur. Ce phénomène de la surreprésentation des hommes, des notables et des ecclésiastiques a également été signalé dans diftérents lieux de pèlerinage en France ${ }^{15}$. Mais ces disproportions ne doivent pas faire oublier le fait le plus important: la croyance au miracle était commune aux laïcs comme aux clercs, et à tous les niveaux de la population.

\section{B. Les saints invoqués}

Parmi les thaumaturges invoqués par la population figuraient des saints recommandés depuis toujours par l'Église (comme la Sainte Vierge, sainte Anne, saint Joseph, la Sainte Famille) plus des personnes vertueuses, canonisées par le peuple après leur mort avec la bénédiction du clergé: $\mathrm{M}^{\mathrm{gr}}$ de Laval et $\mathrm{M}^{\mathrm{gr}}$ de Lauberivière, le frère Didace Pelletier, récollet d'origine canadienne, Jeanne Leber, Catherine Tekakouita, etc. L'enthousiasme populaire pouvait être éphémère: la confiance subite manifestée aux trois religieuses de l'Hôpital-Général ne semble pas avoir duré plus d'une quinzaine de jours, mais jamais, dans la région de Québec, on ne vit le clergé s'opposer au culte d'un saint non reconnu par l'Église.

Le nombre de miracles attribué à chaque saint peut être considéré comme un indice de sa popularité, mais ces données chiffrées ne doivent pas nous faire illusion, certains documents ayant disparu et d'autres étant fort imprécis. Malgré leurs lacunes, les témoignages révèlent que sainte Anne jouissait incontestablement de la plus grande réputation de thaumaturge avec une cinquantaine de miracles à son actif. Le frère Didace venait au second rang. La Vierge, invoquée sous des vocables divers, avait un palmarès moins imposant, mais on lui reconnaissait deux miracles exceptionnels: la victoire sur les Anglais en 1690 et 1710. Tous les autres saints se voyaient attribuer moins de dix miracles chacun, chiffre trop faible ou imprécis pour permettre des comparaisons significatives.

\footnotetext{
15 Jean Delumeau, dir., La mort des pays de Cocagne. Comportements collectifs de la Renaissance à l'âge classique, Paris, 1976, pp. 175 et 179. Hervé Barbin et Jean-Pierre Duteil, «Miracle et pèlerinage au XVII ${ }^{\mathrm{e}}$ siècle», Revue d'histoire de l'Église de France. LXI, 167 (juillet-décembre 1975), p. 253.
} 
Si on dresse la liste des personnes canonisées par le peuple après avoir vécu au Canada, on constate que les deux sexes sont à peu près également représentés, mais que presque toutes appartiennent à l'état religieux (11 sur 13). En outre, les deux seules laïques, Jeanne Leber et Catherine Tekakouita, menaient une vie très proche de celle des religieuses: la première, recluse, la seconde, vouée à la virginité. L'idée que le peuple se faisait de

la sainteté se rapprochait donc beaucoup du modèle proposé par l'Église qui considérait la vie religieuse comme supérieure au mariage ou au célibat, et la virginité comme la forme la plus parfaite de la chasteté.

\section{Tableau 4}

\section{Personnes «canonisées» par le peuple}

Frère Didace

$\mathrm{M}^{\mathrm{gr}}$ de Lauberivière

Jean de Brébeuf, s.j.

$\mathrm{M}^{\mathrm{gr}}$ de Laval

Jacques Marquette, s.j.

Jacques de Lamberville, $s . j$.

D.R. Thaumur de La Source
Catherine Tekakouita

Jeanne Leber

Catherine de Saint-Augustin

Trois religieuses de l'Hôpital-Général de Québec.

Tous ces thaumaturges étaient polyvalents: les gens leur demandaient les faveurs les plus diverses. Dans les dangers de la navigation, on invoquait habituellement sainte Anne, mais aussi la Vierge, saint Joseph et saint Augustin. Le clergé présentait l'époux de Marie comme le patron de la bonne mort, mais les gens s'adressaient aussi à la Vierge pour demander cette dernière faveur.

Afin de mettre plus de chances de leur côté, certains malades invoquaient plusieurs saints en même temps. Après quoi, les différents narrateurs faisaient ressortir l'efficacité du saint qu'ils préféraient. En 1665, par exemple, un aveugle, Jean Adam, se voua à sainte Anne et à Notre-Damede-Lorette. Thomas Morel, curé de Sainte-Anne-du-Petit-Cap, écrivit que cet aveugle avait eu trois éclairs de vision lors d'un pèlerinage dans cette église $^{16}$. Les jésuites desservant la mission de Lorette insistèrent sur le fait qu'il retrouva entièrement la vue dans cette chapelle au terme de sa deuxième neuvaine ${ }^{17}$. Marie de l'Incarnation, enfin, qui prônait la dévo-

\footnotetext{
16 Thomas Morel, «Miracles arrivés en l'église de Sainte-Anne du Petit-Cap coste de Beaupray en Canada, 1662-67», Archives du Séminaire de Québec (désormais ASQ), Paroisses diverses, $\mathrm{n}^{\circ} 72,5^{\mathrm{e}}$ miracle.

${ }^{17} R J$ (1665), t. 5, p. 18.
} 
tion à la Sainte Famille, affirma que «sainte Anne ne voulut pas lui accorder cette grâce qu'elle savait être réservée à l'invocation de la sainte Famille. On [le] ram[ena] donc à Québec devant l'autel de cette famille Sainte où la vue lui fut rétablie» ${ }^{18}$.

\section{Les différents types de miracles}

Parmi les faveurs que les gens demandaient au ciel et obtenaient de façon miraculeuse, les guérisons venaient au premier rang ( 96 cas), suivies des sauvetages maritimes ( 27 cas), des interruptions d'incendies ( 6 cas) et autres fléaux naturels. Au nombre des faveurs spirituelles figuraient la bonne mort (3 cas), la conversion des païens et des hérétiques.

Les maladies et infirmités guéries miraculeusement n'étaient pas toujours identifiées ou décrites avec précision, mais quelques-unes revenaient vraiment plus souvent que d'autres. Il s'agit de la cécité, de la paralysie et des troubles entrainant l'incapacité de voir ou de marcher. Voulant donner une idée d'ensemble des miracles survenus à Sainte-Anne-du-Petit-Cap, Marie de l'Incarnation mettait d'ailleurs l'accent sur ces deux maux: «On y voit marcher les paralitiques, les aveugles recevoir la vue, et les malades de quelque maladie que ce soit recevoir la santé» ${ }^{19}$. Le même phénomène a été constaté dans plusieurs sanctuaires, en France et en Orient, ce qui amène les historiens à conclure d'abord à la fréquence de ces deux infirmités, puis à une imitation inconsciente du modèle évangélique ${ }^{20}$.

Les maladies féminines comme les grossesses difficiles, les fièvres puerpérales et autres maladies «qu'on ne peut décemment nommer» revenaient fréquemment dans les récits. Les enfants, eux, étaient le plus souvent guéris de «fâcheuses descentes», terme qui désigne probablement une hernie.

Les personnes qui recouraient ainsi à l'aide du ciel avaient généralement épuisé sans succès les remèdes humains. L'une d'entre elle, Magdeleine Duplessis, a retracé les différentes étapes de sa guérison. Souffrant des suites de la petite vérole, elle s'adressa d'abord aux détenteurs officiels

18 Marie de l'Incarnation, Correspondance, éd. Dom Guy Oury, Abbaye de Saint-Pierre, Solesmes, 1971, p. 755, 30 sept. 1665.

${ }^{19}$ Ibid.

${ }^{20}$ H. Barbin et J.-P. Duteil, «Miracle et pèlerinage», p. 251. J. Delumeau, Mort des pays de Cocagne, p. 180. Pierre-André Sigal, «Maladie, pèlerinage et guérison au XVII ${ }^{\text {e }}$ siècle, les miracles de saint Gibrien à Reims", Annales. Économies. Sociétés. Civilisations, 24,6 (novembre-décembre 1969), p. 1528. 
du savoir médical: le chirurgien Alavoine qui lui fit une saignée, puis le pharmacien jésuite Boispineau qui lui donna des eaux minérales. Elle se tourna ensuite vers le révérend père Dunainie (?) qui lui suggéra des cataplasmes, et enfin vers une guérisseuse, la dame Biron, mais toujours sans succès. En désespoir de cause, elle mit toute sa confiance en Dieu et lui attribua sa guérison finale ${ }^{21}$.

Les malades adressaient habituellement eux-mêmes leurs prières à Dieu ou aux saints. Faisaient exception à cette règle les très jeunes enfants et les malades inconscients: leurs parents ou les religieuses qui les soignaient demandaient alors leur guérison à leur place.

Lors des accidents de navigation, les personnes qui réquéraient l'aide du ciel pouvaient être tout aussi bien des marins aguerris comme Iberville $^{22}$, des administrateurs importants comme Talon ${ }^{23}$ ou Tracy ${ }^{24}$, des religieuses ou de simples habitants.

La plupart du temps, les naufragés s'adressaient à sainte Anne. Cela dépendait probablement du fait qu'entre 1625 et 1665, Sainte-Anned'Auray, en Bretagne, était un lieu de pèlerinage très fréquenté par les marins ${ }^{25}$. Les immigrants bretons ont donc pu introduire cette dévotion. D'autre part, on sait que la côte de Beaupré fut d'abord peuplée par des colons venus du Perche, province de France où sainte Anne jouissait également d'une grande popularitée ${ }^{26}$. Ces deux facteurs ont donc pu contribuer à l'implantation de cette dévotion en Amérique.

Les incendies constituaient un autre fléau terriblement redouté par la population mal armée pour les combattre. Quand un sinistre de ce genre éclatait dans une ville, on vit à maintes reprises un prêtre se précipiter sur les lieux en apportant le Saint-Sacrement. À chaque fois, des gens affirmèrent avoir vu les flammes s'apaiser ou le vent les détourner. Ces miracles de type eucharistique se sont multipliés en France après le concile de

${ }^{21}$ ASQ, Paroisses diverses, $n^{\circ} 70$.

22 Lettre du père Gabriel Marest au père de Lamberville, 1694. Lettres édifiantes et curieuses écrites des missions étrangères, Lyon, 1819, p. 9.

${ }^{23}$ Marie-Françoise Juchereau et Hélène Duplessis, Annales de l'Hôtel-Dieu de Québec (désormais $A H D Q$ ), Montréal, Presses de Garden City, 1939, pp. 167-168.

24 Journal des Jésuites (désormais $J J$ ), Montréal, Éd. François-Xavier, 1973, p. 348 et Auguste Gosselin, L'Église du Canada, Québec, Demers, 1890, t. 2, p. 480.

25 Jean Delumeau, Mort des pays de Cocagne, pp. 170-183.

${ }^{26}$ Marcel Trudel, Histoire de la Nouvelle France, III, La seigneurie des Cent Associés, Montréal, Fides, 1979, pp. 130-132. Françoise Siguret, Perche Canada, dans la série Cahiers percherons, $\mathrm{n}^{\circ} \mathrm{XX}, 1964$, p. 35. 
Trente ${ }^{27}$. Dans ce cas précis, ce sont visiblement les ecclésiastiques qui s'efforçaient d'orienter la dévotion populaire vers le Christ.

À l'occasion, certains catholiques demandaient à Dieu de concrétiser ce passage du Notre-Père: «Donnez-nous aujourd'hui notre pain quotidien». Les annales de plusieurs communautés religieuses renferment le récit d'une mystérieuse multiplication des pains. L'événement se déroulait habituellement de la façon suivante: alors que les religieuses se trouvaient piesquiuc à boùt de ressourices, elles donnaient l'un de leurs derniers pains à un pauvre. Après quoi, le reste de leurs provisions se multipliait de façon incompréhensible pour répondre aux besoins de la communauté. Les narratrices présentaient ces faits comme une récompense de leurs activités charitables ou de la confiance en la divine Providence manifestée par leur sainte fondatrice.

Derniers cas d'interventions mystérieuses: les présumées apparitions de la Vierge Marie. En 1665, quand une femme inconnue vint prendre soin des enfants de Marie Haslé pendant que celle-ci assistait à la réunion de la confrérie de la Sainte-Famille, plusieurs personnes crurent que cette gardienne était la Vierge elle-même ${ }^{28}$. Et lorsqu'un vieillard presque aveugle s'égara en se rendant à l'assemblée de la Congrégation mariale, il appela aussitôt la Vierge à son aide et se dit ensuite convaincu que la personne qui l'avait secouru était la mère de $\operatorname{Dieu}^{29}$. Des récits de ce genre devaient se multiplier dans la tradition folklorique québécoise au $\mathrm{XIX}^{\mathrm{e}}$ et au $\mathrm{XX}^{\mathrm{e}}$ siècles, confirmant l'image maternelle et bienfaisante que le peuple se faisait de la Vierge.

La majorité des miracles consistait en bienfaits matériels. Certains phénomènes spirituels, comme les conversions, étaient aussi considérés comme des miracles mais rarement rendus publics parce que confinés au secret du confessionnal. L'une des principales faveurs spirituelles demandées consistait à faire une «bonne mort», c'est-à-dire, selon les critères de l'époque, une mort précédée d'une agonie consciente qui permettait au mourant de recevoir les derniers sacrements pour faciliter l'entrée au ciel. À deux reprises, des parents ou des religieuses invoquèrent la Vierge et saint Joseph pour obtenir qu'un malade tombé dans le coma reprenne connaissance assez longtemps pour se confesser et, apparemment du moins, être sauvé. Mais aucun document ne mentionne des résurrections d'enfants mort-nés ou l'existence de "chapelles à répit». Ces chapelles, habituelle-

\footnotetext{
${ }^{27}$ Marcel Bernos, «Miracles chez les Servites en Provence à l'époque moderne», Revue d'histoire de la spiritualité, $\mathrm{n}^{\circ} 49$ (1973), p. 256.

${ }^{28} R J$ (1665), t. 5, p. 19.

${ }^{29} M^{g r}$ de Saint-Vallier et l'Hôpital-Général, Québec, Darveau, 1882, pp. 149-151.
} 
ment dédiées à la Vierge, étaient fort nombreuses en France. On y amenait les enfants morts sans baptême pour demander à la Vierge de leur redonner vie pendant quelques instants, et on profitait ensuite de ce «répit» pour les baptiser et leur ouvrir ainsi les portes du ciel ${ }^{30}$. Les autorités ecclésiastiques ne cachaient pas leur hostilité à cette pratique qui ne semble pas s'être transplantée dans la colonie.

L'abjuration de deux protestants hospitalisés à l'Hôtel-Dieu de Québec fut également jugée quasi miraculeuse par les narratrices. Dans le premier cas, on attribua la conversion à l'intercession du père de Brébeuf (Catherine de Saint-Augustin avait fait avaler au malade un fragment de ses reliques) $)^{31}$, dans le deuxième, à l'intervention de la Vierge Marie car le patient s'était converti subitement au passage d'une procession le jour de l'Assomption ${ }^{32}$. Ajoutons que dans les deux cas, les protestants avaient été soumis aux exhortations des religieuses et de l'aumônier et qu'ils avaient pu être touchés par la sollicitude de leurs garde-malades et l'atmosphère de piété qui régnait à l'Hôtel-Dieu.

\section{Moyens de recours}

Les personnes qui demandaient au ciel un secours extraordinaire pouvaient se contenter de formuler leur requête sous forme d'une prière mentale. Mais la plupart employaient des moyens plus visibles, plus concrets comme les voeux, les pèlerinages, les neuvaines, ou des moyens tout à fait matériels comme les images et les reliques.

Dans les cas de danger vraiment pressant, comme un naufrage ou une maladie mortelle, les personnes menacées n'avaient évidemment pas la possibilité d'entreprendre une neuvaine ou un pèlerinage, mais elles pouvaient très bien promettre de les accomplir une fois que Dieu et le saint qu'elles invoquaient les auraient tirées d'affaire. D'où la fréquence des voeux dans le processus miraculeux.

Le clergé insistait sur l'obligation d'exécuter fidèlement ces promesses. Un cas de démence survenu à l'Hôtel-Dieu fut interprété comme une punition d'un voeu non accompli ${ }^{33}$. Et les laïcs partageaient cette convic-

30 Marcel Bernos, «Réflexions sur un miracle à l'Annonciade d'Aix-en-Provence. Contribution à l'étude des sanctuaires «à répit», Annales du Midi, 82 (1970), pp. 5-20.

${ }^{31} A H D Q$, p. 148.

32 Registre des malades de l'Hôtel-Dieu de Québec, vol. 4, p. 640. Archives Nationales de Québec.

${ }^{33} A H D Q$, pp. 21, 45-46. 
tion. Toutes les personnes qui avaient promis de faire dire des messes à Sainte-Anne-du-Petit-Cap exigeaient qu'elles soient célébrées à cet endroit, même si le curé débordé par la besogne aurait voulu en faire dire une partie dans les paroisses voisines ${ }^{34}$. Certaines personnes craignaient même d'être punies si elles ne manifestaient pas convenablement leur reconnaissance: la prochaine fois, le saint ne les exaucerait $\mathrm{pas}^{35}$.

L'acquittement d'un voeu prenait parfois la forme d'un tableau, appclé cx-voto, qui illustrait la faveur obtenue et le saint invoqué. Parmi la vingtaine d'ex-voto qui datent du Régime français, la majorité s'adressent à sainte Anne et représentent des accidents de la navigation.

Les pèlerinages pouvaient être faits pour accomplir un voeu ou pour demander une faveur, les gens croyant peut-être que le saint qu'ils invoquaient les entendrait mieux s'ils priaient sur son tombeau ou dans un sanctuaire qui abritait ses reliques. Sous le Régime français, Sainte-Anne-duPetit-Cap était le lieu de pèlerinage le plus fréquenté et de nombreux miracles s'y produisaient. L'affluence augmentait particulièrement le 26 juillet, jour de la fête patronale, ce qui entraînait une forte augmentation des revenus de la fabrique mais aussi des désordres, comme les excès de boisson et les bagarres, que le clergé s'efforçait vainement d'enrayer. Parmi les innombrables pèlerins, on distinguait des personnages importants comme M. de Tracy $^{36}$ et le gouverneur Denonville ${ }^{37}$, des Indiens venus de la mission de Lorette $^{38}$ et de nombreux navigateurs. Bref, des gens de tous les milieux sociaux.

Des pèlerinages moins nombreux avaient également lieu à des sanctuaires dédiés à la Vierge comme Notre-Dame-des-Anges, Notre-Damede-Foy et Notre-Dame-de-Lorette. À ce dernier endroit se produisit un incident particulièrement éclairant au sujet des relations existant entre le clergé post-tridentin et le peuple. Le pape avait accordé une indulgence plénière à tous ceux qui se confessaient et communiaient dans cette chapelle le jour de l'Annonciation. En 1701, l'édifice subit quelques modifications et du coup, certaines personnes s'imaginèrent que l'indulgence avait été anéantie. $\mathrm{M}^{\mathrm{gr}}$ de St-Vallier s'empressa alors de confirmer la validité de cette dernière en exprimant son désir «d'obvier à toutes les peines et

\footnotetext{
34 Texte écrit au verso d'une lettre de Pierre de Mimiac, vicaire-général, annonçant sa visite, 7 février 1733. Archives de la basilique Sainte-Anne-de-Beaupré (désormais ABSAB), PA-2, 1032-1.

35 «Actes du Frère Didace», Le Canada français, 4 (1891), p. 271.

$36 \mathrm{JJ}$, pp. 343 et 348 .

${ }^{37}$ Livre de comptes de Sainte-Anne du Petit-Cap, I, mars 1686. ABSAB.

38 Église du village huron de l'Ancienne-Lorette, plaque commémorative.
} 
scrupules que les personnes ignorantes et mal instruites pouvaient avoir» ${ }^{39}$. Ce fait révèle un trait caractéristique de la dévotion populaire: l'importance que les gens du peuple accordaient au support matériel de la dévotion, dans le cas présent la chapelle. L'évêque, de son côté, se montra capable de s'adapter à la mentalité populaire, de se mettre au niveau de ses ouailles pour les instruire.

Cet attachement, au sens propre du terme, au support matériel de la dévotion se manifestait également dans l'utilisation des reliques et des images. Ces objets de piété, les gens les portaient sur eux pour s'attirer une protection, les appliquaient sur un membre malade dans l'espoir d'un soulagement, et parfois même, ils les avalaient comme pour en absorber la vertu sacrale. Ces pratiques étaient communes aux clercs et aux laïcs. Des religieuses de l'Hôtel-Dieu ${ }^{40}$ et un missionnaire jésuite ${ }^{41}$ firent absorber à des malades des fragments de reliques dissous dans de l'eau en demandant au ciel leur guérison. Ils voyaient sans doute dans ces procédés de simples moyens de propitiation, comme l'Église l'enseignait, mais il y a lieu de se demander si les gens du peuple, «ignorants et mal instruits», n'attribuaient pas un pouvoir magique à l'objet lui-même.

En certaines circonstances, on prêtait même aux statues le pouvoir de se déplacer. Une statue de la Vierge transportée à la Nouvelle-Lorette revenait obstinément vers l'Ancienne ${ }^{42}$; une autre, laissée à l'Hôtel-Dieu, se tournait continuellement vers l'Hôpital-Général ${ }^{43}$. Les habitants de l'Ancienne-Lorette en tirèrent la conclusion que la Vierge voulait rester dans son ancienne résidence, opinion confirmée par $\mathrm{M}^{\mathrm{gr}}$ de Laval, tandis que les religieuses de l'Hôpital-Général furent convaincues que le Ciel approuvait leur fondation. Dans les deux cas, les gens se montraient persuadés que la Vierge animait vraiment les images la représentant.

Tout en enseignant, par la parole et l'exemple, la légitimité du recours aux saints thaumaturges, les ecclésiastiques impliqués dans le processus miraculeux, veillaient toujours à rappeler au peuple que l'auteur des miracles était Dieu lui-même et non le saint intercesseur. «Je demandai ma

\footnotetext{
${ }^{39} \mathrm{M}^{\mathrm{gr}}$ de Saint-Vallier, 1701, «Confirmation d'une indulgence plénière accordée à la chapelle de Lorette». Archives de l'Archevêché de Québec, 12A, registre A, pp. 693-694.

$40 A H D Q$, pp. $182-183$ et 148 .

${ }^{41} R J$ (1672), t. 6, pp. 33-34.

42 Céline et Charlotte Gros-Louis, La chapelle huronne de Lorette, 1730-1980, Village des Hurons, 1980, p. 91.

${ }^{43} M^{g r}$ de Saint-Valier et l'Hôpital-Général, p. 114.
} 
guérison à Dieu par les mérites de son serviteur [le frère Didace]» ${ }^{44}$, écrivit $\mathrm{M}^{\mathrm{gr}}$ de Saint-Vallier. L'abbé Morel de son côté faisait ressortir tous les cas où une guérison se produisait durant la messe: un malade guérit pendant qu'on célèbre la messe pour lui, un aveugle recouvre la vue au moment de l'élévation de l'hostie, une paralytique se met à marcher pour aller communier ${ }^{45}$.

L'édification du peuple était l'autre but poursuivi par le clergé. $\mathbf{M}^{\mathrm{gr}}$ de Sảint-Vallier sounhaitait quie l'ôn imitc les vertus du frère Didace ${ }^{46}$ et que l'on demande à sainte Anne des faveurs pour l'âme et non seulement pour le $\operatorname{corps}^{47}$. De cette manière, les ecclésiastiques s'efforçaient de hausser le niveau spirituel de la dévotion populaire, mais il est impossible de savoir jusqu'à quel point le peuple les suivait dans cette voie.

\section{E. Recours collectifs}

Dans les périodes de calamités publiques (guerres, épidémies et disettes), c'est de façon collective que le peuple implorait l'aide de Dieu et de ses saints. Mais dans ces circonstances, le clergé tenait un discours particulièrement culpabilisant: Brodant sur un thème probablement vieux comme le monde, il présentait tous les fléaux naturels comme les châtiments de Dieu irrité par les péchés des hommes. La seule façon d'apaiser son courroux était une conversion sincère suivie de prières publiques. Repentant ou pas, le peuple participait volontiers aux processions et payait les grandmesses sans barguigner. Les saints les plus invoqués dans ces occasions étaient saint Roch et saint Sébastien, les patrons des paroisses et les saints dont on possédait les reliques. Quelquefois, des témoins affirmaient que l'aspersion d'eau bénite ou l'exposition du Saint-Sacrement avait été suivie par l'anéantissement des chenilles, par une ondée bienfaisante ou par un dégel depuis longtemps attendu.

Dans certaines circonstances, le clergé prononçait même les exorcismes contre les insectes nuisibles aux biens de la terre. Cette cérémonie présentait à coup sûr l'avantage de calmer l'angoisse des gens qui croyaient

\footnotetext{
${ }^{44} \mathrm{M}^{\mathrm{gr}}$ de Saint-Vallier, «Miracle obtenu par l'intercession du frère Didace», 9 juin 1716, dans Mandements des évêques de Québec (désormais $M E Q$ ) publiés par H. Têtu et C.O. Gagnon, Québec, Côté, 1887, I, pp. 487-488.

${ }^{45}$ Thomas Morel, «Miracles arrivés en l'église de Sainte-Anne du Petit-Cap», ASQ, Paroisses diverses, $\mathrm{n}^{\circ} 72$.

${ }^{46} M E Q$, I, p. 488.

${ }^{47} \mathrm{M}^{\mathrm{gr}}$ de Saint-Vallier, 20 juin 1726, dans Guy Plante, « $\mathrm{M}^{\mathrm{gr}}$ de Saint-Vallier et la dévotion à sainte Anne», Revue d' histoire de l'Amérique française, 24,3 (décembre 1970), p. 408.
} 
voir des sorciers à l'origine de ces fléaux ${ }^{48}$. Tout en admettant la possibilité théorique que les hommes puissent être tourmentés dans leurs biens par maléfices ou infestations, $\mathbf{M}^{\mathrm{gr}}$ de Saint-Vallier avertissait ses diocésains de ne pas soupçonner leurs voisins de ces méfaits, mais de recourir plutôt aux prières de l'Église qui possède le pouvoir de les aider ${ }^{49}$. Si le phénomène de la chasse aux sorcières fut remarquablement rare en NouvelleFrance, c'est peut-être justement parce que les moyens de recours proposés par l'Église dans les moments difficiles suffisaient à sécuriser la population.

\section{Conclusion}

L'étude de la croyance au miracle dans le gouvernement de Québec, sous le Régime français, révèle bien des points communs dans le comportement des catholiques du Canada et de la mère patrie: surreprésentation des mêmes groupes de personnes parmi les bénéficiaires des miracles, similitude des types de miracles et des maladies guéries le plus fréquemment, importance commune accordée aux lieux et aux objets de dévotion (images et reliques), importance si grande qu'elle nous porte à croire, comme bien des historiens français, que le peuple attribuait un pouvoir magique ou une vertu sacrale à l'objet représentant le saint ${ }^{50}$. Mais le sentiment qui domine dans la croyance au miracle, c'est l'impérieux besoin de recours d'une population désarmée devant la maladie et les catastrophes naturelles.

Face à ces attentes des populations, le clergé des deux pays savait faire preuve de compréhension. Il inscrivit des fêtes agraires au calendrier liturgique, prévit des prières et des bénédictions pour les biens de la terre, voire des exorcismes contre les maléfices susceptibles de détruire le bétail et les récoltes. Comme le peuple éprouvait de la difficulté à formuler une prière purement mentale, le clergé approuva l'utilisation des images et des reliques.

En même temps qu'il répondait aux besoins du peuple, le clergé s'efforça de relever le niveau de sa vie religieuse. Établissant un lien entre les malheurs publics et les péchés des hommes, il incita ces derniers à mieux

\footnotetext{
48 Marie-Aimée Cliche, La religion populaire dans le gouvernement de Québec, pp. 96-108. ${ }^{49} \mathrm{M}^{\mathrm{gr}}$ de Saint-Vallier, Rituel du diocèse de Québec, Paris, Simon Langlois, 1703, pp. 487-488.

50 Alphonse Dupront, «Pèlerinages et lieux sacrés», Encyclopedia Universalis, 12, p. 733. Dominique Julia, "Discipline ecclésiastique et culture paysanne aux $\mathrm{XVII}^{\mathrm{e}}$ et XVIII ${ }^{\mathrm{e}}$ siècles», dans La Religion populaire, pp. 202-203.
} 
se conformer aux lois de Dieu et de l'Église, et à demander des biens spirituels et non seulement matériels. Inlassablement, le clergé répéta que Dieu seul était le dispensateur de tous les biens tandis que le saint jouait le rôle d'un intermédiaire.

Le désir d'éclairer la religion du peuple amenait le clergé à observer d'un oeil attentif la fréquentation des lieux de culte. Et c'est ici qu'apparaît la principale différence dans la religion populaire en France et dans la colonie. Dans la mère patrie, le clergé post-tridentin s'évertuait à combattre des superstitions très anciennes qui sentaient le paganisme, à jeter hors des églises les statues indécentes, à interdire la fréquentation nocturne des chapelles rurales et des fontaines miraculeuses, à faire la chasse aux saints douteux ${ }^{51}$. Il ne semble pas avoir eu autant de problèmes dans la colonie. Ici, pas de chapelles à répit, pas de fontaines miraculeuses, pas de cultes aberrants comme celui de saint Guinefort, un lévrier réputé guérisseur d'enfants ${ }^{52}$. Sans doute était-il plus facile de contrôler l'implantation de la dévotion dans un pays neuf que de déraciner des coutumes médiévales. Le clergé ne relâchait pas sa vigilance pour autant, surveillant en particulier les chapelles rurales ${ }^{53}$, mais ce contrôle était préventif plutôt que répressif.

Dans l'ensemble, la croyance populaire au miracle ne s'opposait pas à l'enseignement du clergé qui d'ailleurs ne trouvait pas de plaintes à formuler à ce sujet. Mais n'allons pas en conclure que cette conformité découlait de l'obéissance des habitants à leurs curés. Quand les autorités religieuses dictaient une ligne de conduite qui s'opposait vraiment au comportement populaire, elles avaient le plus grand mal à faire respecter leur volonté: le mode de célébration des fêtes patronales, surtout celle de sainte Anne, en constitue la meilleure preuve ${ }^{54}$. Dans le cas des miracles, s'il existe une grande conformité entre le comportement des laïcs et l'enseignement dispensé par le clergé, c'est parce qu'au fond, ce dernier répondait aux attentes de la population.

\footnotetext{
${ }^{51}$ Dominique Julia, «Discipline ecclésiastique», pp. 199-209.

52 Jean-Claude Schmitt, Le Saint Lévrier. Guinefort, guérisseur d'enfants depuis le XIII' siècle, Paris, Flammarion, 1979.

${ }^{53}$ Marie-Aimée Cliche, La religion populaire dans le gouvernement de Québec, pp. 27-29.

${ }^{54}$ Ibid ., pp. 33-37.
} 